\title{
Illuminating cellular biochemistry: fluorogenic chemogenetic biosensors for biological imaging
}

\author{
Fanny Broch, ${ }^{[a]}$ and Arnaud Gautier*[a,b] \\ [a] F. Broch, Prof. Dr. A. Gautier \\ Sorbonne Université, École normale supérieure, PSL University, CNRS, Laboratoire des biomolécules, LBM, 75005 Paris, France \\ arnaud.gautier@sorbonne-universite.fr \\ [b] Prof. Dr. A. Gautier \\ Institut Universitaire de France
}

\begin{abstract}
Cellular activity is defined by the precise spatiotemporal regulation of various components, such as ions, small molecules or proteins. Studying cell physiology consequently requires the optical recording of these processes, notably by using fluorescent biosensors The recent developments of various fluorogenic systems greatly expanded the palette of reporters to be included in these sensors design. Fluorogenic reporters consist in a protein or RNA tag that can complex either an endogenous or a synthetic fluorogenic dye (socalled fluorogen). The intrinsic nature of these tags, along with the high tunability of their cognate chromophore provide interesting features such as far-red to near-infrared emission, oxygen independence or unprecedented color versatility. These engineered photoreceptors, self-labelling proteins, or non-covalent aptamers and protein-tags were rapidly identified as promising reporters to observe biological events. This review focuses on the new perspectives they offer to design unique and innovative biosensors, thus pushing the boundaries of cellular imaging.
\end{abstract}

\section{Arnaud Gautier studied Chemistry at the École Normale Supérieure de Lyon, where he received a $\mathrm{PhD}$ in Chemistry in 2005. After postdocs in Chemical Biology, first at the École Polytechnique Fédérale of Lausanne and then at the MRC laboratory of Molecular Biology in Cambridge, he joined the École Normale Supérieure in Paris in 2010 as Assistant Professor. Since 2019, Arnaud Gautier is full professor of Chemistry at Sorbonne University in Paris, where he develops a research program on the development of chemogenetic tools for biological imaging.}

Fanny Broch graduated in Molecular and Biological chemistry from the École Polytechnique Fédérale de Lausanne in 2017. She is currently a $2^{\text {nd }}$ year PhD student, working under the supervision of Arnaud Gautier on the development of fluorogenic chemogenetic biosensors for various applications in Cell Biology.

\section{Introduction}

The pioneering discovery of the green fluorescent protein (GFP) and its development into a fluorescent reporter has been a landmark for the investigation of cellular structure and physiology. ${ }^{[1]}$ The subsequent use of genetically encoded probes and their improvements have tremendously widened our understanding of living matter over the last two decades. ${ }^{[2]}$ These fluorescent markers represent powerful and reliable tools for imaging proteins of interest, cellular components or even structures as complex as the neuronal circuits in a brain. ${ }^{[3]}$

Deciphering complex cellular processes requires synthetic or genetically encoded biosensors able to detect small metabolites, enzyme activity or specific recognition events. Genetically encoded biosensors are commonly designed by coupling a fluorescent reporter together with a sensing unit (e.g. environment-sensitive domain, analyte-binding domain or enzyme substrate domain) that undergoes a conformational change upon input signal. They can generally be categorized in two classes: intensiometric and ratiometric biosensors. Intensiometric sensors consist in a single fluorescent reporter with fluorescence emission properties sensitive to the conformational or environmental change undergone by its coupled sensing unit. Ratiometric biosensors on the other hand consist in a sensing unit conjugated with two fluorophores that form a Förster Resonance Energy Transfer (FRET) pair: upon biological event, a change in the FRET efficiency is observed due to a change in the position and orientation of the two fluorophores.

Genetic encoding allows the targeting of GFP-based biosensors with absolute specificity in various cell types and subcellular localizations. Recent reviews show how the development of such biosensors greatly helped deciphering biological processes, by allowing the observation of various cellular signaling pathways with high spatial and temporal resolution. ${ }^{[4]}$ The performance and reliability of these reporter systems allow their use not only in cells, but also in high-throughput metabolic screenings. ${ }^{[5]}$

The calcium ion sensor GCaMP nicely illustrates how genetically encoded intensiometric biosensors can be designed by coupling GFP-like fluorescent proteins with analyte recognition domains (Figure 1A). GCaMP relies on the $\mathrm{Ca}^{2+}$-dependent interaction between calmodulin (CaM) and the peptide $\mathrm{M} 13$, linked to each terminus of circularly permuted EGFP: brightness of the fluorescent protein is restored upon the calcium-induced interaction between CaM and M13. ${ }^{[6]}$ Many improved calcium indicators followed, optimizing the sensitivity, reliability, color and brightness. ${ }^{[7,8]}$ Fluorescent proteins have also been essential for the design of ratiometric biosensors, by insertion of a sensing domain between two fluorescent proteins, acting as FRET donor and acceptor. (Figure 1B). This strategy was illustrated by the early development of Cameleons, a family of $\mathrm{Ca}^{2+}$ sensors also based on CaM/M13. ${ }^{[9]}$ Similar designs notably allowed the visualization of kinase activity by inserting a substrate peptide sequence between the FRET pair. ${ }^{[10]}$ Sensing of small molecules 

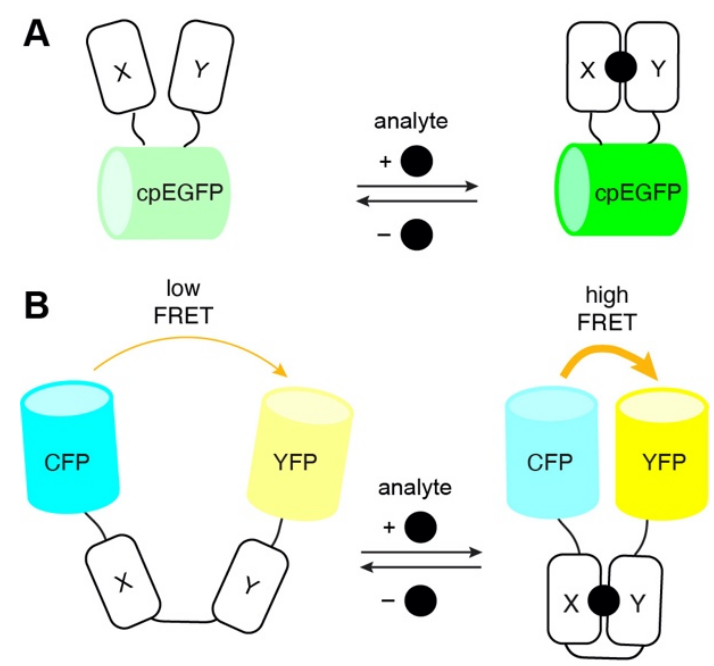

C

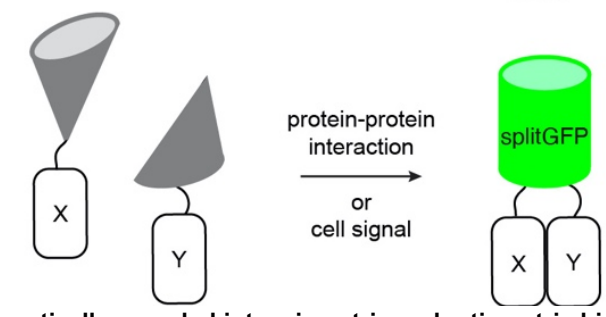

Figure 1. Genetically encoded intensiometric and ratiometric biosensors. (A) General design of intensiometric biosensor based on single fluorescent protein (cPEGFP: circularly permutted enhanced green fluorescent protein). (B) General design of FRET-based biosensors (CFP: cyan fluorescent protein (FRET donor), YFP: yellow fluorescent protein (FRET acceptor)). (C) Bimolecular fluorescence complementation (BIFC) assays for the detection of protein-protein interactions or cell signal.

such as glutamate was also allowed by insertion of periplasmic binding proteins: these two-domains receptors were particularly useful in the development of the glutamate sensor GluSnFR and its improved versions for neurotransmission imaging. ${ }^{[11,12]}$

Finally, fluorescent proteins were also elegantly used to design reporters of protein-protein interactions (PPIs). Splitting fluorescent proteins in two complementary fragments allowed the design of bimolecular fluorescence complementation (BiFC) assays for the detection of PPI, relying on the reconstitution of a functional fluorescent reporter when the two fragments are brought in close proximity (Figure 1C). ${ }^{[13]} \mathrm{A}$ subsequent tripartite split-GFP allowed to reach higher in vivo signal-to-noise ratio. ${ }^{[14]}$

These different systems are well characterized and commonly used to design reliable assays both in vitro and in vivo; yet they present certain limitations that arise from the use of GFPlike fluorescent proteins. The relatively large size of fluorescent proteins can drastically modify folding of the sensing domain, as well as its affinity for its cognate analyte, in a way that is hardly predictable. The observation of dynamic processes can also be impeded by the lag time between event occurrence and actual signal observation, due to the delay necessary for protein folding and chromophore maturation. The latter being dependent on the presence of oxygen, the use of these biosensors is also restricted to aerobic conditions. Finally, regarding split fluorescent proteins, despite their many applications and improvements (particularly in terms of solubility and decreased self-assembly) their reconstitution is irreversible: consequently, they cannot be used for the study of both association and dissociation of two interacting proteins. ${ }^{[15]}$
The frontiers of biosensing were further pushed with the initial developments of the self-labeling proteins SNAP-tag ${ }^{[16]}$, HaloTag ${ }^{[17]}$, and CLIP-tag[18], which enable the selective anchoring of tunable synthetic probes. In addition to their essential role as labeling tools, chemogenetic sensors can be constructed in situ, taking advantage of the genetically encoded tag to target environment- or cation-sensitive probes with absolute specificity. This way $\mathrm{Ca}^{2+}$ transients, ${ }^{[19,20]}$ dynamics of other biologically relevant cations, ${ }^{[21-23]}$ as well as microviscosity, ${ }^{[2]}$ could be precisely evaluated at different sub-cellular localizations. Combination of HaloTag with a voltage sensitive rhodopsin also yielded an electrochromic FRET voltage sensor for the imaging of action potentials in various organisms. ${ }^{[25]}$ Moreover, the general design of Snifits, ${ }^{[26,27]}$ where SNAP-tag is labelled with a bifunctional molecule containing both a FRET acceptor and an affinity ligand for the analyte-binding protein, was applied to the imaging of several neurotransmitters ${ }^{[28-30]}$ (Figure 2). This approach allowed the combination of SNAP-tag with various donors such as a fluorophore conjugated to the orthogonal HaloTag ${ }^{[31]}$, or even with a bioluminescent luciferase. ${ }^{[32]}$

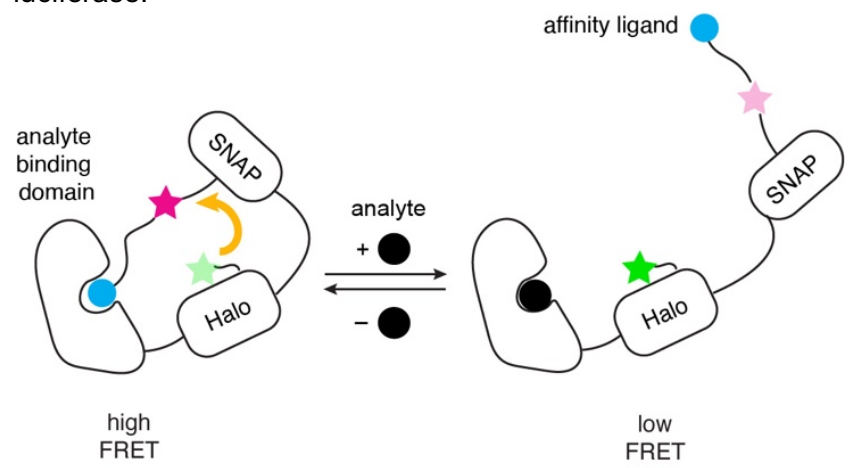

Figure 2. Schematic illustration of the Snifit biosensors.

Recently, biosensor design have taken a new turn with the development of chemogenetic systems composed of proteins or RNA aptamers that bind and activate the fluorescence of fluorogenic dyes (so-called fluorogens), which are otherwise dark when free (Figures 3 and $\mathbf{4}$ ). These fluorescent hybrid reporters make use of natural or synthetic fluorogens, each having their own advantages. Natural fluorogens are endogenously present in cells and thus do not need to be delivered (if present at sufficient concentrations). Synthetic fluorogens, on the other hand, can be tuned and equipped with new properties by molecular engineering. Moreover, they need to be exogenously applied, offering new possibilities to create original labeling protocols. The underlying mechanisms, advantages and general applications of these fluorogen-activating systems have been thoroughly described in recent reviews. ${ }^{[33,34]}$ Here we focus on the new opportunities these novel chemogenetic hybrid reporters offer for the design of innovative biosensors enabling to observe and study biological processes in new ways.

\section{Chemogenetic optical reporters and biosensors based on natural fluorogens}

Various chemogenetic optical reporters were designed from chromoproteins that incorporate natural fluorogenic chromophores such as flavin or bilins as prosthetic groups. These 
A

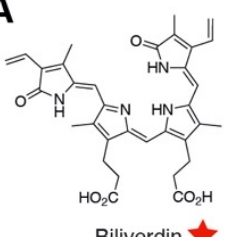

.

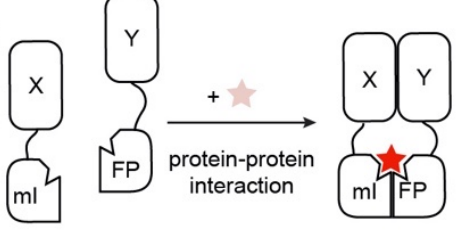

C

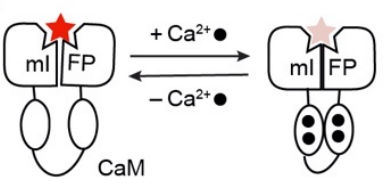

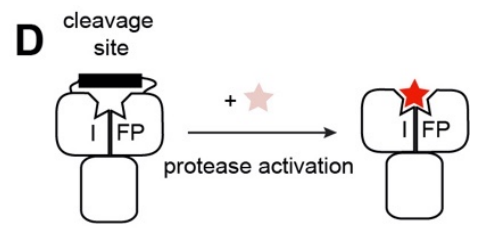

G
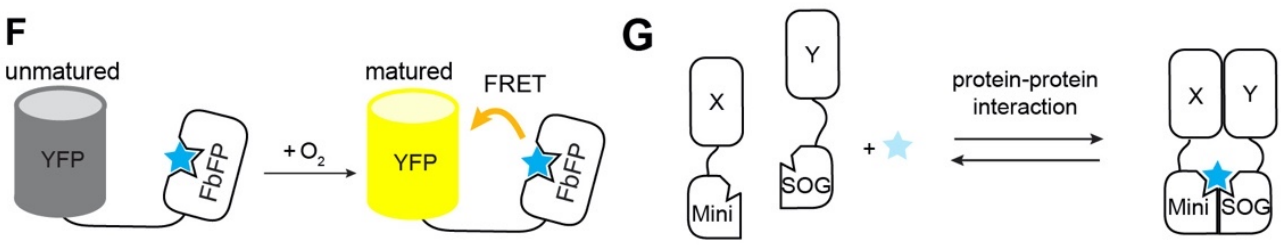

$\mathrm{FMN}$

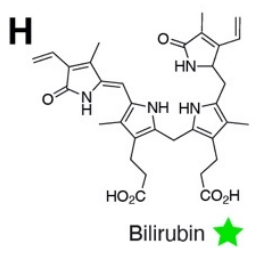

I

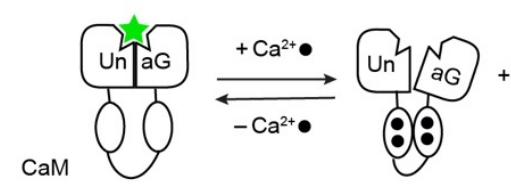

J
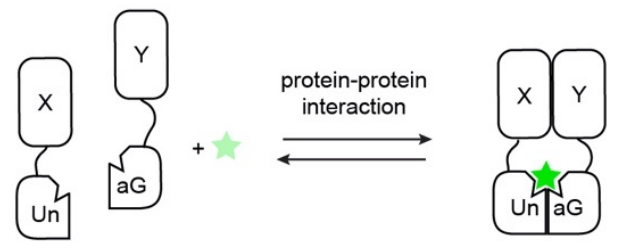

Figure 3. Fluorescent chemogenetic biosensors based on natural fluorogens. (A) Chemical structure of the endogenous fluorogen biliverdin (BV). (B) iSplit: irreversible reconstitution of mIFP upon protein-protein interaction allows covalent BV binding, thus fluorescence activation. (C) NIR-GECO1: Conformational change of the calmodulin sensing module upon calcium binding yields fluorescence inactivation of the complexed BV. (D) iProtease: release of the biliverdin binding site after protease cleavage allows covalent binding of BV, thus fluorescence activation. (E) Chemical structure of the endogenous fluorogen flavin mono-nucleotide FMN. (F) FluBO: YFP chromophore maturation, thus FRET efficiency, depends on molecular oxygen levels. (G) Split miniSOG: reversible reconstitution assembly upon protein-protein interactions allows non-covalent binding of $\mathrm{FMN}$, and thus fluorescence activation $(\mathrm{H})$ Chemical structure of the endogenous fluorogen bilirubin (BR). (I) BReleaCa: conformational change of calmodulin upon $\mathrm{Ca}^{2+}$ binding reduces UnaG affinity for BR, thus fluorescence intensity. ( $\mathrm{J}$ ) uPPI: reversible reconstitution of UnaG upon protein-protein interaction allows non-covalent binding of $\mathrm{BR}$, thus fluorescence activation.

chromoproteins are often weakly fluorescent because they evolved to maximize the efficiency of their natural photocycle. Protein engineering allowed to enhance their fluorescence properties by impairing their natural photocycle.

\subsection{Biliverdin-based optical reporters and biosensors}

Far-red and near-infrared fluorescent optical reporters were engineered from bacterial phytochromes. These photoreceptors sense far-red light through photoisomerization of a covalently attached biliverdin (BV) chromophore (Figure 3A) and function as light driven signal transducers. $B V$ is an endogenous and ubiquitous molecule that is non-fluorescent when free but can fluoresce near-infrared light when immobilized. Near-infrared fluorescent markers are highly desirable for both imaging and biosensing applications. They enable to reduce phototoxicity from excitation light, to dramatically decrease auto-fluorescence from living samples, and to image in deeper tissues because of reduced scattering. Near-infrared fluorescence was however difficult to attain with GFP-like fluorescent proteins: one of the redder one, mNeptune, reaches an emission maximum at $650 \mathrm{~nm}$. The first infrared optical reporter was designed by suppression of the PHY domain of the bacteriophytochrome of Deinococcus radiodurans, and introduction of key mutations in the immediate environment of $\mathrm{BV}$ in the remaining PAS-GAF domains to prevent BV photoisomerization and lock it into an emissive conformation. ${ }^{[35]}$ The resulting mutant IFP1.4 displays excitation and emission maxima at 684 and $708 \mathrm{~nm}$, respectively, and allowed imaging both in vitro and in living mice. Improved brightness and photostability were obtained with iRFP (ex/em $690 / 713 \mathrm{~nm}$ ), a near-infrared variant similarly evolved from a different bacteriophytochrome $(\mathrm{BphP})$ scaffold, that reached observable brightness level without exogenously applied biliverdin. ${ }^{[36]}$

These enhanced properties made iRFP a suitable candidate for in vivo studies of PPIs with BiFC. iRFP was split between its PAS and GAF domains, generating two fragments that could be then respectively fused to each interacting partner (Figure 3B). This infrared split system (iSplit) formed a highly stable and irreversible complex, that enabled visualization of the FKBP-FRB interaction up to 36 hours after rapamycin injection in living mice. ${ }^{[37]}$ The irreversibility of the complementation can allow the integration of transient or low abundant interactions in a cumulative manner, however it prevents imaging of dynamic processes involving dissociation of a protein-protein complex. A reversible split IFP was engineered shortly after, by testing fluorescence brightness and dissociation capacity of fragments from several IFP1.4 splitting sites. The interaction of PKA catalytic and regulatory subunits and their CAMP-induced dissociation could thus be observed in both mammalian cells and yeast. ${ }^{[38]}$

The above mentioned IFPs inherited a tendency to dimerize at high concentrations, as the BphPs they originate from are multimeric. This dimeric nature may hinder further development of infrared reporters, as it can interfere with the dynamics of the observed process. Consequently, a monomeric mIFP was engineered from a monomeric truncated $\mathrm{BphP}$, only retaining the PAS-GAF domains. ${ }^{[39]}$ A near-infrared $\mathrm{Ca}^{2+}$ indicator for optical imaging (NIR-GECO1) was obtained by insertion of the calcium binding domain calmodulin into mIFP (Figure 3C). ${ }^{[40]}$ The fluorescent properties and $\mathrm{Ca}^{2+}$-dependent response of this biosensor were optimized by random mutagenesis and screening of the connection sites between the different domains. NIRGECO1 showed an inverse response to $\mathrm{Ca}^{2+}$ transients, that 
could be slightly improved by addition of exogenous biliverdin in cultured neurons. This sensor was shown to be useful in multiplexed imaging experiments, up to four colors (three indicators, together with a FRET indicator and a single-FP sensor). Recently an analogous biosensor was engineered from the small monomeric GAF-FP, which was rationally designed and evolved to be the minimal size biliverdin-binding single domain. ${ }^{[1]}$ Insertion in GAF-FP of the calcium-dependent pair CaM/M13 resulted in the near-infrared $\mathrm{Ca}^{2+}$ indicator GAF-CaMP2. Screening of different insertion sites, randomization of two aminoacids linkers at connecting sites, and further mutagenesis of the initial scaffold were necessary to adjust the affinity and the dynamic range of the final design. Contrary to its predecessor this indicator gives a positive response, nevertheless it needs to be fused to the superfolding sfGFP to be correctly expressed in mammalian cells, reaching 1.2-fold larger molecular size than NIR-GECO1. GAF-CaMP2 enabled ratiometric measurement of intracellular $\mathrm{Ca}^{2+}$ transients, and allowed their visualization in three different organelles when expressed with two other GFPbased calcium sensors. ${ }^{[42]}$

Infrared fluorogenic reporters were also used for the monitoring of cell apoptosis. Circular permutation of IFP, connecting the PAS and GAF domains with a motif recognized by a specific protease, displaced the catalytic cysteine away from the biliverdin complexation cavity, thus impeding fluorogen binding. iProtease design consequently relied on the reconstitution of functional IFP after protease cleavage, leading to an observable fluorescence increase (Figure 3D). This strategy applied to a caspase reporter iCasper, successfully reported apoptosis in neurons, during morphogenesis of Drosophila embryo and during tumorigenesis. ${ }^{[43]}$

A brighter set of monomeric infrared reporters, miRFPs, were evolved from the PAS-GAF domains of the Rhodopseudomonas palustris Bacteriophytochrome protein $R p B p h P 1$, by random mutagenesis and selection of the brightest mutants in mammalian cells, in parallel with screening in bacteria. ${ }^{[44]}$ This way three spectrally distinct miRFP variants were developed, and were then applied to various biosensors. A BiFC system based on their split version (miSplits) successfully reported two different PPIs in the same cell, and was also used as an RNA reporter when fused to two proteins interacting with RNA with high affinity. Interestingly, the smallest NIR FP to date $(17 \mathrm{kDa})$ was recently derived from a biliverdin-binding cyanobacteriochrome (CBCR) scaffold. ${ }^{[45]}$ miRFP670nano was successfully used as a FRET pair donor in fully NIR sensors for different kinases activities, that could be coupled in cell experiments with optogenetic tools. Furthermore, by fusion of distinct miRFPs to two different proteins subject to cell-cycle dependent degradation at opposite phases, a far-red version of the previously reported fluorescence ubiquitination-based cell cycle indicator (FUCCI) ${ }^{[46]}$ was developed and applied for detection of proliferation status of cells in living mice ${ }^{[44]}$ Following the same strategy, a near-infrared $\mathrm{FUCCl}$ was also developed based on the small ultra-red FP (smURFP) ${ }^{[47]}$, an IFP evolved from allophycocyanin $\alpha$-subunit (Trichodesmium erythraeum) to form a bright covalent complex with biliverdin.

\subsection{Flavin-based optical reporters and biosensors}

One challenge that can remarkably be addressed by engineering fluorogenic systems is the visualization of biological processes under anaerobic conditions. Indeed, the strict oxygendependence of GFP-like proteins for their chromophore maturation restricts their use to aerobic environments. This limitation prompted the development of fluorescent reporters that could be applied both in the presence and absence of oxygen.

The light oxygen voltage (LOV) domain of bacterial photoreceptors were modified to generate cyan-green fluorescent reporters that non-covalently bind flavin mononucleotide (FMN) (Figure 3E). Only retaining the photoactive LOV domain of the receptor, where the reactive cysteine was substituted by a nonreactive alanine, significantly improved the brightness of the complex formed with FMN. The biosynthesis of this natural chromophore does not require oxygen, and contrary to biliverdinbinding IFPs, FMN-binding fluorescent proteins (FbFPs) are not limited by chromophore endogenous supply. ${ }^{[48-50]}$ An hypoxia FRET biosensor (fluorescent protein-based biosensor for oxygen, FluBO) rapidly exploited this oxygen independence, by coupling a donor FbFP to the oxygen-sensitive yellow fluorescent protein (YFP) as acceptor. ${ }^{[51]}$ This system offers a direct ratiometric readout of molecular oxygen levels. Indeed, hypoxic conditions prevent maturation of YFP chromophore and most of the fluorescent signal originates from the FbFP, while oxygen supply immediately increases fluorescence from YFP (Figure 3F). Similarly, the dimeric flavoenzyme lipoamide dehydrogenase was fused to mCherry to obtain a FRET sensor for intracellular $\mathrm{NAD}^{+} / \mathrm{NADH}$ ratio. ${ }^{[52]}$ The flavoprotein reduction alters fluorescence from the bound cofactor flavin adenine dinucleotide, altering FRET efficiency, thus offering a ratiometric evaluation of the NAD/NADH redox state. This FRET biosensor design could in principle be generalized, although it remains restricted to the limited variety of sufficiently bright flavoenzymes in their oxidized form.

Interestingly, the FbFP variant $\mathrm{iLOV}^{[53]}$ showed intrinsic affinity for $\mathrm{Cu}^{2+}$, with high specificity in regard of other metal ions. This property allowed the use of iLOV as a reliable $\mathrm{Cu}^{2+}$ biosensor: at physiological $\mathrm{pH}$, its fluorescence is reversibly quenched up to $80 \%$ upon $\mathrm{Cu}^{2+}$ binding, supposedly from coordination of the metal ion to $\mathrm{FMN}$-interacting residues in the binding site. ${ }^{[54]}$ Another metal cation, $\mathrm{Mn}^{3+}$, could be probed by a flavoprotein mutant in which tyrosine analogues were incorporated. ${ }^{[55]}$

Interestingly, the role of the LOV cofactor FMN is not limited to fluorogenicity, but also includes photosensitizer properties by generation of reactive oxygen species (ROS) without the need for any exogenous cofactor. These ROS are short-lived and phototoxic, inducing irreversible damage on cellular components. The mini Singlet Oxygen Generator (miniSOG) takes advantage of these characteristics to localize single proteins of interest or cellular structures with high resolution by correlated light and electron microscopy (CLEM). Generation of singlet oxygen ${ }^{1} \mathrm{O}_{2}$ under blue-light illumination allowed the local polymerization of osmiophilic polymers that can be detected by electron microscopy. ${ }^{[56]}$ Directed evolution yielded an improved miniSOG2 that allowed highly specific cell ablation of single-neurons in the Drosophila larvae. ${ }^{[57]}$ This photosensitizer function was also improved by site-directed mutagenesis, resulting in a singlet oxygen photosensitizing protein SOPP $3,{ }^{[58]}$ and used as a genetically encoded antimicrobial agent that proved effective against Gram-positive and negative bacteria. ${ }^{[59]}$ With the aim of using CLEM to study intracellular PPIs, a miniSOG variant with improved solubility was split at a viable circular permutation position (Figure 3G). ${ }^{[6]}$ This development led to two soluble protein fragments that could efficiently and reversibly reassemble, offering a way to study protein-protein interactions such as the neurotoxic assemblies of $\alpha$-synuclein by CLEM. 


\subsection{Bilirubin-based optical reporters and biosensors}

Hypoxia can also be probed by different sensors based on the UnaG reporter, a natural protein that fluoresces green light when complexing the endogenous bilirubin (Figure $3 \mathrm{H}$ ) ${ }^{[61]}$ UnaG shows higher fluorescence quantum yield than FbFP, and was consequently a promising candidate to improve molecular oxygen probes under both aerobic and anaerobic conditions. One strategy consisted in genetically encoding destabilized UnaG with a promoter showing positive hypoxia-specific activity, inducing expression at low oxygen levels. ${ }^{[62]}$ Destabilization allows an efficient protein turnover, resulting in decreasing fluorescence under oxygen supply. Targeted incorporation of cysteines forming a disulfide bridge also allowed to monitor cellular redox state and hypoxia with reduced/oxidized UnaG (roUnaG) in mammalian cells and in bacteria with addition of exogenous bilirubin. ${ }^{[63]}$

UnaG was elegantly used to design biosensors by conditioning bilirubin unbinding to the recognition of a given analyte. A $\mathrm{Ca}^{2+}$-sensor based on UnaG, BReleaCa, ${ }^{[64]}$ was developed by inserting the $\mathrm{Ca}^{2+}$-binding protein $\mathrm{CaM}$ close to the bilirubin binding site (Figure 31). The resulting sensor gives an onoff response to $\mathrm{Ca}^{2+}$ increase, due to a significantly reduced affinity of UnaG for bilirubin in the $\mathrm{Ca}^{2+}$-bound state of the hybrid protein.

A

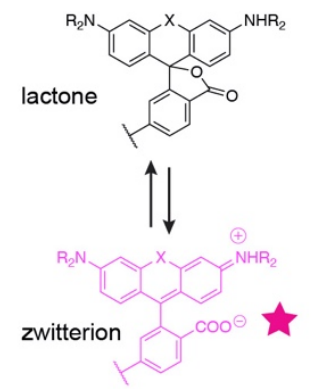

B

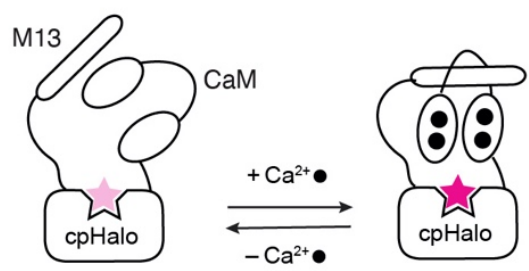

C

\section{Chemogenetic optical reporters and biosensors based on synthetic fluorogens}

The recent development of chemogenetic optical reporters based on synthetic fluorogens opened new prospects for the design of optical biosensors. The use of synthetic fluorogens rather than natural ones allows the generation of fluorogenicity through various activation mechanisms, and the tuning of fluorogen properties by molecular engineering.

rhodamine

D<smiles>CC1=N/C(=C/c2cc(F)c(O)c(F)c2)C(=O)N1C</smiles>

$\mathbf{E}$

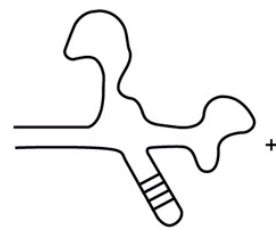

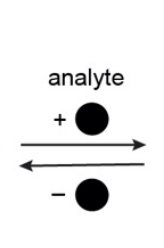

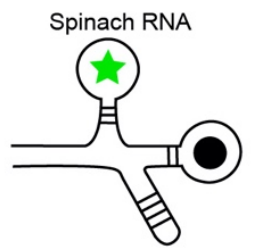

Analyte binding aptamer
$\mathbf{F}$

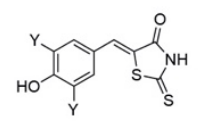

HBR analogs

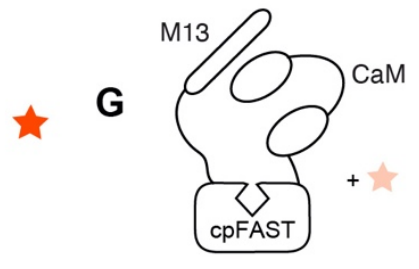

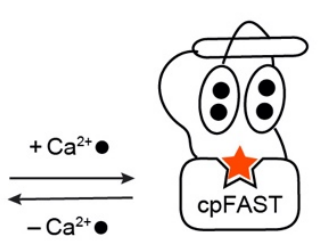
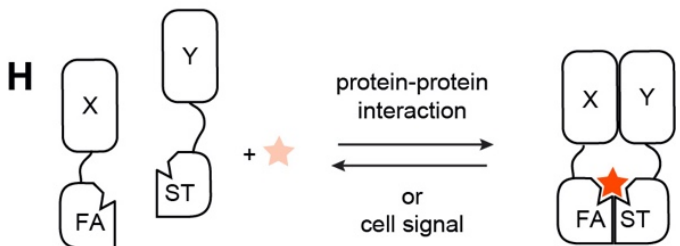

Figure 4. Fluorescent chemogenetic biosensors based on synthetic fluorogens. (A) Chemical structure of synthetic fluorogenic rhodamines. (B) HaloCaMP: upon $\mathrm{Ca}^{2+}$ binding, conformational change of the sensing module CaM/M13 yields fluorescence activation of the rhodamine dye covalently linked to a circularly permuted (cp) HaloTag. (C) HArcLight: conformational change of a voltage-sensitive domain yields fluorescence activation of the rhodamine dye covalently linked to a circularly permuted HaloTag. (D) Chemical structure of the synthetic fluorogen 3,5-difluoro-4-hydroxybenzylidene imidazolinone (DFHBI) (E) RNA aptamers: Conformational change of the sensing module upon analyte complexation allows non-covalent binding of DFHBI, thus fluorescence activation. (F) Chemical structure of the synthetic fluorogenic HBR analogs. (G) FAST-based $\mathrm{Ca}^{2+}$ sensor: conformational change of the sensing module CaM/M13 increases affinity for the HBR analog, thus fluorescence intensity. (H) split FAST: reversible reconstitution of FAST upon assembly of the sensing module allows non-covalent binding of the HBR analog, thus fluorescence activation.

\subsection{Covalent fluorogenic labeling}

Recent efforts have been undertaken for the development of fluorogenic chromophores able to covalently and orthogonally label the previously mentioned protein tags SNAP-tag ${ }^{[16]}$,
HaloTag ${ }^{[17]}$ and CLIP-tag ${ }^{[18]}$. Silicon-rhodamine (SiR) derivatives for instance are found in their dark spirolactone form in solution, but in their bright zwitterionic form when bound to a protein tag (Figure 4A). ${ }^{[6]}$ Highly cell-permeable fluorogenic analogs were 
newly obtained by replacing the carboxyl group by an electronpoor amide group, in equilibrium with its spirolactam form. ${ }^{[67]}$ Similarly, fluorogenic Janelia Fluor (JF) dyes derived from carbofluorescein and carborhodamine were developed and applied to super-resolution microscopy, as well as single-particle tracking experiments. ${ }^{[68-70]}$ These strategies avoid conjugation of the fluorophore with an intramolecular quencher to create fluorogenecity, and different functionalization allowed the development of cyan to near-infrared fluorogens for multicolor imaging. ${ }^{[71]}$ The cationic aromatic fluorophores "channel dyes" also show large fluorescence increase upon binding to HaloTag, due to cation- $\pi$ interaction with a tryptophan benzene ring. ${ }^{[2,73]}$

Self-labeling proteins can be elegantly engineered to obtain fluorogenic hybrid indicators. A destabilized variant of HaloTag prone to aggregation (AgHalo) was notably engineered to report on impaired proteostasis induced by cellular stress. ${ }^{[7,75]}$ AgHalo aggregation was detected using a fluorogenic ligand that fluoresces only when AgHalo forms insoluble aggregates. Fusion of AgHalo to a protein of interest enables the study of its stressinduced aggregation. An orthogonal SNAP-tag based system was developed as an addition to the aggregation tag method (AggTag), ${ }^{[76]}$ allowing the simultaneous detection of two different proteins' aggregation. Very recently, a circularly permuted (cp)HaloTag version was elegantly included in the design of fluorescent chemogenetic biosensors, combining both protein engineering and fine-tuning of the coupled fluorophore. ${ }^{[77]}$ The fluorescence of JF dyes can be enhanced by increased interactions with surface residues of HaloTag, allowing the creation of highly sensitive indicators through conformational coupling to a sensing domain. Coupling of cpHaloTag with. calcium-binding domain and voltage-sensitive domain allowed the creation of calcium and voltage indicators (Figure 4B,C), which were successfully used in cultured rat hippocampal neurons using diverse and interchangeable environment-sensitive JF dyes, further illustrating the flexibility of hybrid reporters.

Systems that directly integrate fluorogenicity in their initial design were recently developed. The cellular retinoic acid binding protein II (CRABPII) ${ }^{[78]}$ and the closely related human Cellular Retinol Binding Protein II (hCRPBII) ${ }^{[79]}$ were engineered into red fluorescent reporters by introducing a lysine residue in their binding cavity to allow the formation of an iminium with aldehydecontaining merocyanine dye precursors. Engineering of hCRPBII combined with the use of a julolidine retinal analog further allowed the development of a ratiometric $\mathrm{pH}$ sensor. ${ }^{[00]}$ The sensor takes advantage of the sensitivity of the bound chromophore to the electrostatic environment within the binding cavity. The absorption profile changes as a function of the protonation state of a carboxylic side chain in proximity of the bound iminium. Unfortunately, the julolidine retinal analog was not membranepermeable, preventing cellular evaluation.

The photoactive yellow protein (PYP) was also engineered into a small self-labeling protein tag, taking advantage of its ability to react with hydroxycynamoyl and coumaryl thioester derivatives. ${ }^{[81]}$ Fluorogenic ligands were obtained coupling a fluorescent moiety and a quencher, ${ }^{[82]}$ or using environmentsensitive fluorogens that only display high fluorescence intensity in the low-polar cavity of the protein tag. ${ }^{[83]}$ Engineering of both fluorogens and PYP mutants led to enhanced brightness and labeling kinetics. ${ }^{[84-87]} A$ sensor for methylated-DNA was obtained by fusing a methyl-CpG binding domain (MBD) with PYP. Labeling with an oxazole yellow (YO) ligand that strongly fluoresces upon DNA-binding allowed to track DNA-methylation during mitosis with good signal-to-noise ratio. ${ }^{[88]}$
Optical reporters relying on covalent tethering of a fluorogen allowed imaging of various biological events. Biosensing was achieved by signal integration, or fluorescence intensity change of a synthetic indicator anchored to a genetically encoded tag. In addition to that, engineering of non-covalent fluorogenic systems greatly expanded the palette of reporters that could be incorporated in the design of biosensors, particularly suitable for monitoring dynamic and reversible processes.

\subsection{Non-covalent fluorogenic labeling}

Among semi-synthetic fluorogenic systems, RNA aptamers are one remarkable alternative to GFP-like FPs, as they can adopt geometrical conformations suitable for small molecules binding. The Spinach RNA aptamer nicely illustrates how short RNA sequences can serve as scaffold to complex synthetic fluorogens such as DFHBI (Figure 4D) with high specificity and affinities ${ }^{[89]}$. The use of SELEX (Systematic Evolution of Ligands by EXponential enrichment), ${ }^{[00]}$ targeted mutagenesis as well as directed evolution later yielded several RNA-fluorogen reporters such as Spinach2, ${ }^{[91]}$ Broccoli, ${ }^{[92]}$ Mango, ${ }^{[93]}$ the Peppers series ${ }^{[94]}$, Riboglow $^{[5]}$ and o-Coral[96]. These systems display high brightness and thermostability, and together with the expanded fluorogen palette including the NIR and cell-permeable $\mathrm{SiR},{ }^{[97,98]}$ they offer a wide range of emission properties for efficient monitoring of RNA dynamics in living cells. ${ }^{[99]}$

Rational design enabled to make fluorophore binding dependent on the presence of analytes endogenously targeted by RNA structures, notably by riboswitches. In this manner, different riboswitches aptamers showing conformational change upon cyclic dinucleotides binding were inserted in Spinach2, leading to specific sensors for c-di-GMP, c-AMP-GMP and c-di-AMP in bacteria (Figure 4E) ${ }^{[100,101]}$ Applications of cyclic dinucleotides biosensors were extended to both aerobic and anaerobic contexts by rational mutagenesis. ${ }^{[102]}$ Despite this successful phylogeneticbased optimization, biosensors design for various small-molecule analytes is generally facilitated by high-throughput screening methods. In this way, a theophylline biosensor was developed as proof-of-concept combining a droplet-based microfluidic screening with Next Generation Sequencing. ${ }^{[103]}$

Recently, biosensors taking advantage of aptamer reporters' nature were developed to track specific RNAs in living cells. The mirror image aptamer L-Mango was used to image micro-RNAs (miRNA): acting as a blocking strand to an achiral peptide nucleic acid (PNA), it is displaced by overexpressed miRNAs and then properly folds to complex and activate its cognate dye. ${ }^{[104]}$ This study shows that L-aptamers are biocompatible and can selfdeliver to cells by cholesterol conjugation, opening the way for new RNA-based probes for imaging and biosensing.

Another type of semi-synthetic reporters, fluorogen activating proteins (FAPs) derived from single-chain variable fragments of antibodies were selected by directed evolution to specifically bind and strongly activate known fluorogens including Malachite Green (MG) or Thiazole Orange (TO). Such fluorophores and their derivatives offer tunable properties such as cell permeability, far-red emission, ${ }^{[105-108]}$ and biocompatible imaging in living cells, yeast ${ }^{[109,110]}$ and bacteria. ${ }^{[111]}$ Control of labeling densities with far-red emitting MG makes it a suitable candidate for super-resolution microscopy, notably for Binding Activated Localization Microscopy (F-BALM) ${ }^{[112]}$ and single particle tracking. ${ }^{[113]} \mathrm{A}$ Cy5 analogue conjugated to TO, showing both cell-exclusion and $\mathrm{pH}$-sensitivity, yielded a FAP-based biosensor able to follow endocytosis and recycling of a surface protein, while indicating $\mathrm{pH}$ evolution in vesicles by FRET 
emission changes. ${ }^{[14]}$ Receptor trafficking visualization with STED microscopy was possible by fusion of the improved $\mathrm{pH}$ biosensor TrapHic, based on a Cy3 analogue-MG tandem dye. ${ }^{[115]} \mathrm{A}$ similar strategy enabled to follow internalization of the pharmacologically-relevant GABAA receptor, and its localization along the neuronal endosome-lysosome axis. ${ }^{[110]}$ Membraneimpermeant dyes were also used to probe opening of synaptic fusion pores that ensure neuropeptides transmission. ${ }^{[16]}$ Easy diffusion of the small size MG compared to the larger intracellular FAP favors the formation of the fluorescent protein-dye complex inside the synaptic pore, interestingly taking advantage of both components' inherent properties. A FAP-based assay for membrane apposition also made ingenious use of both genetic targeting of membrane proteins and modularity of the fluorogen, by synthetically connecting MG and dimethylindole red (DIR). A more than 10 -fold fluorescence increase from the lower affinity dye MG can be observed at the contact sites between co-cultured cells expressing two different FAPs. ${ }^{[17]}$

FAP-based systems can also act as highly localized photosensitizers with dyes functionalized with heavy atoms, notably by activation of the ROS-generating di-iodinated MG analog. ${ }^{[18]}$ Combined with the different strategies to target such systems to tumor cells ${ }^{[119]}$, this property shows promising potential for targeted photodamage. Moreover, FAPs biosensors do not only rely on the fluorogen versatility: the protein part itself can be engineered to undergo observable conformational change. This was illustrated by insertion of a peptide cleavage sequence adjacent to a minimal glycosylation site, connecting FAP to a blocking domain, that can only be released and allow dye binding in case of defective O-glycosylation. ${ }^{[120]}$

A similar but smaller semi-synthetic reporter, the Fluorescence Activating and Absorption-Shifting Tag (FAST), was recently developed. ${ }^{[121]}$ Evolved from the Photoactive Yellow Protein, the cavity of this $14 \mathrm{kDa}$ protein tag can complex fluorogenic compounds derived from the hydroxybenzylidene rhodanine (HBR) family (Figure $4 \mathrm{~F}$ ) in a highly dynamic and reversible manner. The versatility of HBR derivatives makes FAST a suitable candidate for multicolor imaging in various subcellular compartments. ${ }^{[122,123]}$ Membrane-impermeant fluorogens also allowed visualization and quantification of membrane proteins trafficking. ${ }^{[124]}$ An improved FAST version (iFAST) was obtained by introduction of a single mutation (V107I), and dimerization of this reporter resulted in tandem FAST (td-iFAST) that reached higher brightness values than EGFP and mCherry in vitro. ${ }^{[125]}$ FAST fluorescence being oxygen-independent, it is a reliable reporter for the study of bacterial biofilm formation ${ }^{[126]}$ or in strictly anaerobic conditions such as Clostridium organisms. ${ }^{[127]}$ It was also applied to super-resolution microscopy, taking advantage of the rapid fluorogen exchange for single-particle tracking $^{[128]}$ or sub-diffraction imaging with a conventional microscope using Super-Resolution Radial Fluctuations (SRRF). ${ }^{[129]}$ Interestingly, red-shifted GFP-like fluorogens binding FAST with comparable affinity were recently investigated, and show promising photostability for long-time imaging. ${ }^{[130]}$

FAST proved useful as reporting domain in the development of a multicolor real-time $\mathrm{Ca}^{2+}$ indicator. ${ }^{[131]}$ This strategy relied on the $\mathrm{Ca}^{2+}$-dependent interaction of the sensing module CaM/M13, modifying the reporter affinity for its cognate dye, and consequently the observed brightness (Figure 4G). Noteworthy, this sensor was developed using a circularly permuted version of FAST (cPFAST). The creation of new termini closer to the fluorogen binding pocket made it more sensitive to the presence of $\mathrm{Ca}^{2+}$. Careful control of fluorogen concentration allowed one to observe 2 to 3 -fold fluorescence increase upon $\mathrm{Ca}^{2+}$ transients in living cells. FAST also tolerates splitting at its circular permutation site, and reconstitution of the functional tag is rapid and reversible, opening the way to numerous biosensing applications (Figure 4H). First, split FAST enabled real-time observation of both FKBP/FRB complex formation and dissociation, and this proof-ofconcept was then extended to follow MEK1 and ERK2 interaction upon activation of the MAPK signaling pathway. Split FAST was also applied to the design of a caspase activity biosensor to visualize apoptosis, and each split part was fused as well to either CaM or M13 to generate an intermolecular $\mathrm{Ca}^{2+}$ biosensor. ${ }^{[132]}$ This modularity shows great potential for further development of FAST-based biosensors with highly tunable properties to investigate cellular processes in various organisms.

\section{Conclusions \& perspectives}

The recent and plentiful developments of new fluorogenic reporter systems have opened the way towards new horizons in the field of bioimaging and biosensing. The intrinsic properties of these fluorogenic systems, notably oxygen independence, substantially broaden the range of sensors applications. They also constitute a great addition of bio-orthogonal reporting modules for multiplexed biosensing, applicable to the design of single-reporter indicators, FRET sensors or even BiFC.

Systems binding to endogenous fluorogens offer interesting features notably for deep tissue imaging without the need for delivery. On the other hand, tunable synthetic fluorogens make these systems very valuable for modular multicolor biosensing. They enable to change the reporter emission properties in an easier way than switching fluorescent proteins, that generally requires re-engineering of the entire chimera. Thus, the chromophores color palette expansion has allowed simultaneous observation of various cellular processes

The improvements of screening methods combined with increasing knowledge of structural characteristics allows the combination of rational design and high-throughput optimization, yielding more performant biosensors in a faster timeline.

Engineering of new fluorogenic systems, together with the development of microscopy techniques, allowed unprecedented spatial and temporal resolution. New opportunities for in vivo biosensing and super-resolution microscopy are emerging with the expanding availability of fluorogens with adjustable concentrations for the different systems described.

To conclude, the design of biosensors based on fluorogenic reporters represents a great opportunity to observe and study cellular processes in innovative and original ways. The flexibility of their properties allows to overcome most of the usual limitations encountered in the field of fluorescence microscopy. Because of the very promising perspectives they offer for biological imaging, it is a safe bet to say that fluorogenic chemogenetic biosensors will stand as indispensable tools for cell biologists in the coming years.

\section{Notes}

The authors declare the following competing financial interest: A.G. is co-founder and hold equity in Twinkle Bioscience / The Twinkle Factory, a company commercializing the FAST technology. 


\section{Acknowledgements}

This work was supported by the European Research Council (ERC-2016-CoG-724705 FLUOSWITCH)

Keywords: biosensors $\cdot$ chemogenetic reporters - fluorescent labels $\bullet$ fluorescence imaging $\bullet$ proteins

R. Y. Tsien, Annu. Rev. Biochem. 1998, 67, 509-544.

[2] D. M. Chudakov, M. V. Matz, S. Lukyanov, K. A. Lukyanov, Physiol. Rev. 2010, 90, 1103-1163.

[3] J. Livet, T. A. Weissman, H. Kang, R. W. Draft, J. Lu, R. A. Bennis, J. R. Sanes, J. W. Lichtman, Nature 2007, 450, 56-62.

[4] E. C. Greenwald, S. Mehta, J. Zhang, Chem. Rev. 2018, 118 11707-11794.

[5] Y. Zhao, Q. Hu, F. Cheng, N. Su, A. Wang, Y. Zou, H. Hu, X. Chen, H. Zhou, X. Huang, K. Yang, Q. Zhu, X. Wang, J. Yi, L. Zhu, X. Qian, L. Chen, Y. Tang, J. Loscalzo, Y. Yang, Cell Metab. 2016, 21 777-789.

[6] J. Nakai, M. Ohkura, K. Imoto, Nat. Biotechnol. 2001, 19, 137-141. [7] T.-W. Chen, T. J. Wardill, Y. Sun, S. R. Pulver, S. L. Renninger, A. Baohan, E. R. Schreiter, R. a Kerr, M. B. Orger, V. Jayaraman, L. L. Looger, K. Svoboda, D. S. Kim, Nature 2013, 499, 295-300.

[8] Y. Yang, N. Liu, Y. He, Y. Liu, L. Ge, L. Zou, S. Song, W. Xiong, X. Liu, Nat. Commun. 2018, 9, 1504

[9] A. Miyawaki, J. Llopis, R. Heim, J. M. McCaffery, J. A. Adams, M. Ikura, R. Y. Tsien, Nature 1997, 388, 882-887.

[10] A. Y. Ting, K. H. Kain, R. L. Klemke, R. Y. Tsien, Proc. Natl. Acad. Sci. U. S. A. 2001, 98, 15003-15008.

[11] R. Y. Tsien, FEBS Lett. 2005, 579, 927-932.

[12] S. A. Hires, Y. Zhu, R. Y. Tsien, Proc. Natl. Acad. Sci. 2008, 105 4411-4416.

[13] S. Cabantous, T. C. Terwilliger, G. S. Waldo, Nat. Biotechnol. 2005, 23, 102-107

[14] S. Cabantous, H. B. Nguyen, J. D. Pedelacq, F. Koraïchi, A Chaudhary, K. Ganguly, M. A. Lockard, G. Favre, T. C. Terwilliger, G. S. Waldo, Sci. Rep. 2013, 3, 2854.

[15] M. D. Wiens, R. E. Campbell, Wiley Interdiscip. Rev.: Syst. Biol. Med. 2018, 10

[16] A. Keppler, S. Gendreizig, T. Gronemeyer, H. Pick, H. Vogel, K. Johnsson, Nat. Biotechnol. 2003, 21, 86-89.

[17] G. V. Los, L. P. Encell, M. G. McDougall, D. D. Hartzell, N. Karassina, C. Zimprich, M. G. Wood, R. Learish, R. F. Ohana, M. Urh, D. Simpson, J. Mendez, K. Zimmerman, P. Otto, G. Vidugiris, J. Zhu, A. Darzins, D. H. Klaubert, R. F. Bulleit, K. V. Wood, ACS Chem. Biol. 2008, 3, 373-382.

[18] A. Gautier, A. Juillerat, C. Heinis, I. R. Correa, M. Kindermann, F. Beaufils, K. Johnsson, Chem. Biol. 2008, 15, 128-136.

[19] M. Bannwarth, I. R. Corrêa, M. Sztretye, S. Pouvreau, C. Fella, A Aebischer, L. Royer, E. Ríos, K. Johnsson, ACS Chem. Biol. 2009 4, 179-190.

[20] C. Deo, S. H. Sheu, J. Seo, D. E. Clapham, L. D. Lavis, J. Am Chem. Soc. 2019, 141, 13734-13738.

[21] T. Hirata, T. Terai, H. Yamamura, M. Shimonishi, T. Komatsu, K Hanaoka, T. Ueno, Y. Imaizumi, T. Nagano, Y. Urano, Anal. Chem. 2016, 88, 2693-2700.

[22] Y. Matsui, Y. Funato, H. Imamura, H. Miki, S. Mizukami, K. Kikuchi, Chem. Sci. 2017, 8, 8255-8264.

[23] M. L. Zastrow, Z. Huang, S. J. Lippard, ACS Chem. Biol. 2020, 15, 396-406.

[24] C. Wang, X. Song, L. Chen, Y. Xiao, Biosens. Bioelectron. 2017, 91 313-320

[25] A. Abdelfattah, T. Kawashima, A. Singh, O. Novak, H. Liu, Y. Shuai, Y.-C. Huang, J. Grimm, R. Patel, J. Friedrich, B. Mensh, L. Paninski, J. Macklin, K. Podgorski, B.-J. Lin, T.-W. Chen, G. Turner, Z. Liu, M. Koyama, K. Svoboda, M. Ahrens, L. Lavis, E. Schreiter, Science 2019, 365, 699-704.

[26] H. Farrants, J. Hiblot, R. Griss, K. Johnsson, 2017, 1596, 101-117.

[27] M. A. Brun, R. Griss, L. Reymond, K. T. Tan, J. Piguet, R. J. R. W. Peters, H. Vogel, K. Johnsson, J. Am. Chem. Soc. 2011, 133, 16235-16242.

[28] M. A. Brun, K. T. Tan, R. Griss, A. Kielkowska, L. Reymond, K. Johnsson, J. Am. Chem. Soc. 2012, 134, 7676-7678.

[29] A. Masharina, L. Reymond, D. Maurel, K. Umezawa, K. Johnsson, J. Am. Chem. Soc. 2012, 134, 19026-19034.

[30] A. Schena, K. Johnsson, Angew. Chem. Int. Ed. 2014, 53, 13021305.

[31] O. Sallin, L. Reymond, C. Gondrand, F. Raith, B. Koch, K Johnsson, Elife 2018, 7, e32638.

[32] Q. Yu, L. Xue, J. Hiblot, R. Griss, S. Fabritz, C. Roux, P. A. Binz, D. Haas, J. G. Okun, K. Johnsson, Science 2018, 361, 1122-1126.
[33] A. Gautier, A. G. Tebo, BioEssays 2018, 40, 1800118.

[34] T. Péresse, A. Gautier, Int. J. Mol. Sci. 2019, $20,6412$.

[35] X. Shu, A. Royant, M. Z. Lin, T. A. Aguilera, V. Lev-Ram, P. A. Steinbach, R. Y. Tsien, Science 2009, 324, 804-807.

[36] G. S. Filonov, K. D. Piatkevich, L. M. Ting, J. Zhang, K. Kim, V. V. Verkhusha, Nat. Biotechnol. 2011, 29, 757-761.

[37] G. S. Filonov, V. V. Verkhusha, Chem. Biol. 2013, 20, 1078-1086.

[38] E. Tchekanda, D. Sivanesan, S. W. Michnick, Nat. Methods 2014, 11, 641-644.

[39] D. Yu, M. A. Baird, J. R. Allen, E. S. Howe, M. P. Klassen, A. Reade, K. Makhijani, Y. Song, S. Liu, Z. Murthy, S. Q. Zhang, O. D. Weiner, T. B. Kornberg, Y. N. Jan, M. W. Davidson, X. Shu, Nat. Methods 2015, 12, 763-765.

[40] Y. Qian, K. D. Piatkevich, B. Mc Larney, A. S. Abdelfattah, S. Mehta, M. H. Murdock, S. Gottschalk, R. S. Molina, W. Zhang, Y. Chen, J. Wu, M. Drobizhev, T. E. Hughes, J. Zhang, E. R. Schreiter, S. Shoham, D. Razansky, E. S. Boyden, R. E. Campbell, Nat. Methods 2019, 16, 171-174.

[41] K. A. Rumyantsev, D. M. Shcherbakova, N. I. Zakharova, A. V. Emelyanov, K. K. Turoverov, V. V. Verkhusha, Sci. Rep. 2015, 5 , 18348.

[42] O. M. Subach, N. V. Barykina, K. V. Anokhin, K. D. Piatkevich, F. V. Subach, Int. J. Mol. Sci. 2019, 20, 3488.

[43] T. L. To, B. J. Piggott, K. Makhijani, D. Yu, Y. N. Jan, X. Shu, Proc. Natl. Acad. Sci. U. S. A. 2015, 112, 3338-3343.

[44] D. M. Shcherbakova, M. Baloban, A. V. Emelyanov, M. Brenowitz, P. Guo, V. V. Verkhusha, Nat. Commun. 2016, 7, 12405

[45] O. S. Oliinyk, A. A. Shemetov, S. Pletnev, D. M. Shcherbakova, V. V. Verkhusha, Nat. Commun. 2019, 10, 279

[46] A. Sakaue-Sawano, H. Kurokawa, T. Morimura, A. Hanyu, H. Hama, H. Osawa, S. Kashiwagi, K. Fukami, T. Miyata, H. Miyoshi, T. Imamura, M. Ogawa, H. Masai, A. Miyawaki, Cell 2008, 132, 487498

[47] E. A. Rodriguez, G. N. Tran, L. A. Gross, J. L. Crisp, X. Shu, J. Y. Lin, R. Y. Tsien, Nat. Methods 2016, 13, 763-769.

[48] T. Drepper, T. Eggert, F. Circolone, A. Heck, U. Krauß, J. K. Guterl, M. Wendorff, A. Losi, W. Gärtner, K. E. Jaeger, Nat. Biotechnol. 2007, 25, 443-445.

[49] T. Drepper, R. Huber, A. Heck, F. Circolone, A. K. Hillmer, J. Büchs, K. E. Jaeger, Appl. Environ. Microbiol. 2010, 76, 5990-5994. [50] M. Wingen, J. Potzkei, S. Endres, G. Casini, C. Rupprecht, C. Fahlke, U. Krauss, K. E. Jaeger, T. Drepper, T. Gensch, Photochem. Photobiol. Sci. 2014, 13, 875-883.

[51] J. Potzkei, M. Kunze, T. Drepper, T. Gensch, K. E. Jaeger, J. Büchs, BMC Biol. 2012, 10, 28.

[52] D. A. Hudson, J. L. Caplan, C. Thorpe, Biochemistry 2018, 57 , 1178-1189.

[53] S. Chapman, C. Faulkner, E. Kaiserli, C. Garcia-Mata, E. I. Savenkov, A. G. Roberts, K. J. Oparka, J. M. Christie, Proc. Natl. Acad. Sci. U. S. A. 2008, 105, 20038-20043.

[54] Y. Ravikumar, S. P. Nadarajan, C. S. Lee, J. K. Rhee, H. Yun, J. Microbiol. Biotechnol. 2015, 25, 503-510.

[55] X. Liu, L. Jiang, J. Li, L. Wang, Y. Yu, Q. Zhou, X. Lv, W. Gong, Y. Lu, J. Wang, J. Am. Chem. Soc. 2014, 136, 13094-13097.

[56] X. Shu, V. Lev-Ram, T. J. Deerinck, Y. Qi, E. B. Ramko, M. W. Davidson, Y. Jin, M. H. Ellisman, R. Y. Tsien, PLoS Biol. 2011, 9, 4. K. Makhijani, T. L. To, R. Ruiz-González, C. Lafaye, A. Royant, X. Shu, Cell Chem. Biol. 2017, 24, 110-119.

[58] M. Westberg, M. Bregnhøj, M. Etzerodt, P. R. Ogilby, J. Phys. Chem. 2017, 121, 9366-9371.

[59] F. Hilgers, N. L. Bitzenhofer, Y. Ackermann, A. Burmeister, A Grünberger, K.-E. Jaeger, T. Drepper, Int. J. Mol. Sci. 2019, 20, 4608.

[60] D. Boassa, S. P. Lemieux, V. Lev-Ram, J. Hu, Q. Xiong, S. Phan, M. Mackey, R. Ramachandra, R. E. Peace, S. R. Adams, M. H. Ellisman, J. T. Ngo, Cell Chem. Biol. 2019, 26, 1407-1416.

[61] A. Kumagai, R. Ando, H. Miyatake, P. Greimel, T. Kobayashi, Y Hirabayashi, T. Shimogori, A. Miyawaki, Cell 2013, 153, 1602-1611. R. Erapaneedi, V. V Belousov, M. Schäfers, F. Kiefer, EMBO J. 2016, 35, 102-113.

[63] H. Hu, A. Wang, L. Huang, Y. Zou, Y. Gu, X. Chen, Y. Zhao, Y. Yang, Free Radic. Biol. Med. 2018, 120, 255-265.

[64] Y. Shitashima, T. Shimozawa, T. Asahi, A. Miyawaki, Biochem Biophys. Res. Commun. 2018, 496, 872-879.

[65] T. L. To, Q. Zhang, X. Shu, Protein Sci. 2016, 25, 748-753.

[66] G. Lukinavičius, L. Reymond, K. Umezawa, O. Sallin, E. D’Este, F. Göttfert, H. Ta, S. W. Hell, Y. Urano, K. Johnsson, J. Am. Chem. Soc. 2016, 138, 9365-9368.

[67] L. Wang, M. Tran, E. D’Este, J. Roberti, B. Koch, L. Xue, K. Johnsson, Nat. Chem. 2020, 12, 165-172.

[68] J. B. Grimm, A. J. Sung, W. R. Legant, P. Hulamm, S. M. Matlosz, E. Betzig, L. D. Lavis, ACS Chem. Biol. 2013, 8, 1303-1310.

[69] J. B. Grimm, B. P. English, H. Choi, A. K. Muthusamy, B. P. Mehl, P. Dong, T. A. Brown, J. Lippincott-Schwartz, Z. Liu, T. Lionnet, L. D. Lavis, Nat. Methods 2016, 13, 985-988.

[70] Q. Zheng, A. X. Ayala, I. Chung, A. V. Weigel, A. Ranjan, N. Falco, J. B. Grimm, A. N. Tkachuk, C. Wu, J. Lippincott-Schwartz, R. H. Singer, L. D. Lavis, ACS Cent. Sci. 2019, 5, 1602-1613. 
[71] G. Lukinavičius, K. Umezawa, N. Olivier, A. Honigmann, G. Yang, T. Plass, V. Mueller, L. Reymond, I. R. Corrêa, Z. G. Luo, C. Schultz, E. A. Lemke, P. Heppenstall, C. Eggeling, S. Manley, K. Johnsson, Nat. Chem. 2013, 5, 132-139.

[72] S. A. Clark, V. Singh, D. Vega Mendoza, W. Margolin, E. T. Kool, Bioconjug. Chem. 2016, 27, 2839-2843.

[73] Y. Liu, K. Miao, N. P. Dunham, H. Liu, M. Fares, A. K. Boal, X. Li, X. Zhang, Biochemistry 2017, 56, 1585-1595.

[74] Y. Liu, M. Fares, N. P. Dunham, Z. Gao, K. Miao, X. Jiang, S. S Bollinger, A. K. Boal, X. Zhang, Angew. Chem. Int. Ed. 2017, 56, 8672-8676.

[75] Y. Liu, K. Miao, Y. Li, M. Fares, S. Chen, X. Zhang, Biochemistry 2018, 57, 4663-4674

[76] K. H. Jung, S. F. Kim, Y. Liu, X. Zhang, ChemBioChem 2019, 20, 1078-1087.

[77] C. Deo, A. S. Abdelfattah, H. K. Bhargava, A. J. Berro, N. Falco, B. Moeyaert, M. Chupanova, L. D. Lavis, E. R. Schreiter, bioRxiv 2020, 2020.01.08.898783.

[78] I. Yapici, K. S. S. Lee, T. Berbasova, M. Nosrati, X. Jia, C. Vasileiou, W. Wang, E. M. Santos, J. H. Geiger, B. Borhan, J. Am Chem. Soc. 2014, 137, 1073-1080.

[79] W. Wang, Z. Nossoni, T. Berbasova, C. T. Watson, I. Yapici, K. S. S. Lee, C. Vasileiou, J. H. Geiger, B. Borhan, Science 2012, 338, 1340-1344.

[80] T. Berbasova, S. Tahmasebi Nick, M. Nosrati, Z. Nossoni, E. M. Santos, C. Vasileiou, J. H. Geiger, B. Borhan, ChemBioChem 2018, 19, 1288-1295.

[81] Y. Hori, H. Ueno, S. Mizukami, K. Kikuchi, J. Am. Chem. Soc. 2009, 131, 16610-16611.

[82] Y. Hori, K. Nakaki, M. Sato, S. Mizukami, K. Kikuchi, Angew. Chem Int. Ed. 2012, 51, 5611-5614.

[83] Y. Hori, T. Norinobu, M. Sato, K. Arita, M. Shirakawa, K. Kikuchi, J. Am. Chem. Soc. 2013, 135, 12360-12365.

[84] Y. Hori, S. Hirayama, M. Sato, K. Kikuchi, Angew. Chem. Int. Ed. 2015, 54, 14368-14371.

[85] S. Hirayama, Y. Hori, Z. Benedek, T. Suzuki, K. Kikuchi, Nat. Chem. Biol. 2016, 12, 853-859.

[86] Y. Kamikawa, Y. Hori, K. Yamashita, L. Jin, S. Hirayama, D. M Standley, K. Kikuchi, Chem. Sci. 2016, 7, 308-314.

[87] J. Gao, Y. Hori, T. Shimomura, M. Bordy, J. Hasserodt, K. Kikuchi, Chembiochem 2019, 21, 656-662.

[88] Y. Hori, N. Otomura, A. Nishida, M. Nishiura, M. Umeno, I. Suetake K. Kikuchi, J. Am. Chem. Soc. 2018, 140, 1686-1690.

[89] J. S. Paige, K. Y. Wu, S. R. Jaffrey, Science 2011, 333, 642-646.

[90] C. Tuerk, L. Gold, Science 1990, 249, 505-510.

[91] R. L. Strack, M. D. Disney, S. R. Jaffrey, Nat. Methods 2013, 10 1219-1224.

[92] G. S. Filonov, J. D. Moon, N. Svensen, S. R. Jaffrey, J. Am. Chem. Soc. 2014, 136, 16299-16308.

[93] R. J. Trachman, A. Autour, S. C. Y. Jeng, A. Abdolahzadeh, A. Andreoni, R. Cojocaru, R. Garipov, E. V. Dolgosheina, J. R. Knutson, M. Ryckelynck, P. J. Unrau, A. R. Ferré-D’Amaré, Nat Chem. Biol. 2019, 15, 472-479.

[94] X. Chen, D. Zhang, N. Su, B. Bao, X. Xie, F. Zuo, L. Yang, H. Wang, L. Jiang, Q. Lin, M. Fang, N. Li, X. Hua, Z. Chen, C. Bao, J. $\mathrm{Xu}, \mathrm{W}$. Du, L. Zhang, Y. Zhao, L. Zhu, J. Loscalzo, Y. Yang, Nat. Biotechnol. 2019, 37, 1287-1293.

[95] E. Braselmann, A. J. Wierzba, J. T. Polaski, M. Chromiński, Z. E. Holmes, S. T. Hung, D. Batan, J. R. Wheeler, R. Parker, R. Jimenez, D. Gryko, R. T. Batey, A. E. Palmer, Nat. Chem. Biol. 2018, 14, 964-971.

[96] F. Bouhedda, K. T. Fam, M. Collot, A. Autour, S. Marzi, A Klymchenko, M. Ryckelynck, Nat. Chem. Biol. 2019, 16, 69-76. W. Song, R. L. Strack, N. Svensen, S. R. Jaffrey, J. Am. Chem. Soc. 2014, 136, 1198-1201.

[98] R. Wirth, P. Gao, G. U. Nienhaus, M. Sunbul, A. Jäschke, J. Am. Chem. Soc. 2019, 141, 7562-7571.

[99] L. Truong, A. R. Ferré-D'Amaré, Protein Sci. 2019, 28, 1374-1386.

[100] C. A. Kellenberger, C. Chen, A. T. Whiteley, D. A. Portnoy, M. C. Hammond, J. Am. Chem. Soc. 2015, 137, 6432-6435.

[101] C. A. Kellenberger, S. C. Wilson, J. Sales-Lee, M. C. Hammond, J. Am. Chem. Soc. 2013, 135, 4906-4909.

[102] X. C. Wang, S. C. Wilson, M. C. Hammond, Nucleic Acids Res. 2016, 44, e139.

[103] A. Autour, F. Bouhedda, R. Cubi, M. Ryckelynck, Methods 2019, 161, 46-53.

[104] W. Zhong, J. T. Sczepanski, ACS Sensors 2019, 4, 566-570.

[105] C. Szent-Gyorgyi, B. A. Schmidt, Y. Creeger, G. W. Fisher, K. L. Zakel, S. Adler, J. A. J. Fitzpatrick, C. A. Woolford, Q. Yan, K. V. Vasilev, P. B. Berget, M. P. Bruchez, J. W. Jarvik, A. Waggoner, Nat. Biotechnol. 2008, 26, 235-240.

[106] C. Szent-Gyorgyi, R. L. Stanfield, S. Andreko, A. Dempsey, S. Capek, A. Waggoner, I. A. Wilson, M. P. Bruchez, J. Mol. Biol. 2014, 425, 4595-4613.

[107] B. P. Yates, M. A. Peck, P. B. Berget, Mol. Biotechnol. 2013, 54, 829-841.

[108] C. A. Telmer, R. Verma, H. Teng, S. Andreko, L. Law, M. P. Bruchez, ACS Chem. Biol. 2015, 10, 1239-1246.
[109] J. A. J. Fitzpatrick, Q. Yan, J. J. Sieber, M. Dyba, U. Schwarz, C. Szent-Gyorgyi, C. A. Woolford, P. B. Berget, A. S. Waggoner, M. P. Bruchez, Bioconjug. Chem. 2009, 20, 1843-1847.

[110] J. M. Lorenz-Guertin, M. R. Wilcox, M. Zhang, M. B. Larsen, J. Pilli, B. F. Schmidt, M. P. Bruchez, J. W. Johnson, A. S. Waggoner, S. C Watkins, T. C. Jacob, J. Cell Sci. 2017, 130, 3933-3945.

[111] S. Saurabh, A. M. Perez, C. J. Comerci, L. Shapiro, W. E. Moerner, J. Am. Chem. Soc. 2016, 138, 10398-10401.

[112] Q. Yan, S. L. Schwartz, S. Maji, F. Huang, C. Szent-Gyorgyi, D. S. Lidke, K. A. Lidke, M. P. Bruchez, Chemphyschem 2014, 15, 687695.

[113] S. L. Schwartz, Q. Yan, C. A. Telmer, K. A. Lidke, M. P. Bruchez, D. S. Lidke, ACS Chem. Biol. 2015, 10, 539-546.

[114] A. Grover, B. F. Schmidt, R. D. Salter, S. C. Watkins, A. S. Waggoner, M. P. Bruchez, Angew. Chem. Int. Ed. 2012, 51, 48384842.

[115] L. A. Perkins, Q. Yan, B. F. Schmidt, D. Kolodieznyi, S. Saurabh, M B. Larsen, S. C. Watkins, L. Kremer, M. P. Bruchez, Biochemistry 2018, 57, 861-871.

[116] D. Bulgari, D. L. Deitcher, B. F. Schmidt, M. A. Carpenter, C. SzentGyorgyi, M. P. Bruchez, E. S. Levitan, Proc. Natl. Acad. Sci. 2019, 34, 17039-17044.

[117] D. S. Ackerman, K. V. Vasilev, B. F. Schmidt, L. B. Cohen, J. W. Jarvik, Bioconjug. Chem. 2017, 28, 1356-1362.

[118] J. He, Y. Wang, M. A. Missinato, E. Onuoha, L. A. Perkins, S. C. Watkins, C. M. St Croix, M. Tsang, M. P. Bruchez, Nat. Methods 2016, 13, 263-268

[119] Y. Wang, B. Ballou, B. F. Schmidt, S. Andreko, C. M. St. Croix, S C. Watkins, M. P. Bruchez, Physiol. Behav. 2017, 176, 139-148.

[120] C. Bachert, A. D. Linstedt, Traffic 2013, 14, 47-56.

[121] M.-A. Plamont, E. Billon-denis, S. Maurin, C. Gauron, F. M. Pimenta, C. G. Specht, J. Shi, B. Pan, J. Rossignol, N. Morellet, E. Lescop, Y. Chen, A. Triller, T. Le Saux, L. Jullien, A. Gautier, Proc. Natl. Acad. Sci. 2016, 113, 497-502.

[122] C. Li, M. A. Plamont, I. Aujard, T. Le Saux, L. Jullien, A. Gautier, Org. Biomol. Chem. 2016, 14, 9253-9261.

[123] C. Li, M. A. Plamont, H. L. Sladitschek, V. Rodrigues, I. Aujard, P. Neveu, T. Le Saux, L. Jullien, A. Gautier, Chem. Sci. 2017, 8, 55985605.

[124] C. Li, A. Mourton, M. A. Plamont, V. Rodrigues, I. Aujard, M. Volovitch, T. Le Saux, F. Perez, S. Vriz, L. Jullien, A. Joliot, A Gautier, Bioconjug. Chem. 2018, 29, 1823-1828.

[125] A. G. Tebo, F. M. Pimenta, Y. Zhang, A. Gautier, Biochemistry 2018, 57, 5648-5653.

[126] A. Monmeyran, P. Thomen, H. Jonquière, F. Sureau, C. Li, M. A. Plamont, C. Douarche, J. F. Casella, A. Gautier, N. Henry, Sci. Rep. 2018, 8, 10336.

[127] H. E. Streett, K. M. Kalis, E. T. Papoutsakis, Appl. Environ. Microbiol. 2019, 85, e00622-19.

[128] M. Venkatachalapathy, V. Belapurkar, M. Jose, A. Gautier, D. Nair, Nanoscale 2019, 11, 3626-3632.

[129] E. M. Smith, A. Gautier, E. M. Puchner, ACS Chem. Biol. 2019, 14 1115-1120.

[130] N. V. Povarova, S. O. Zaitseva, N. S. Baleeva, A. Y. Smirnov, I. N. Myasnyanko, M. B. Zagudaylova, N. G. Bozhanova, D. A Gorbachev, K. K. Malyshevskaya, A. S. Gavrikov, A. S. Mishin, M. S. Baranov, Chem. Eur. J. 2019, 25, 9592-9596.

[131] A. G. Tebo, F. M. Pimenta, M. Zoumpoulaki, C. Kikuti, H. Sirkia, M.A. Plamont, A. Houdusse, A. Gautier, ACS Chem. Biol. 2018, 13, 2392-2397.

[132] A. G. Tebo, A. Gautier, Nat. Commun. 2019, 10, 2822. 


\section{Table of Contents}

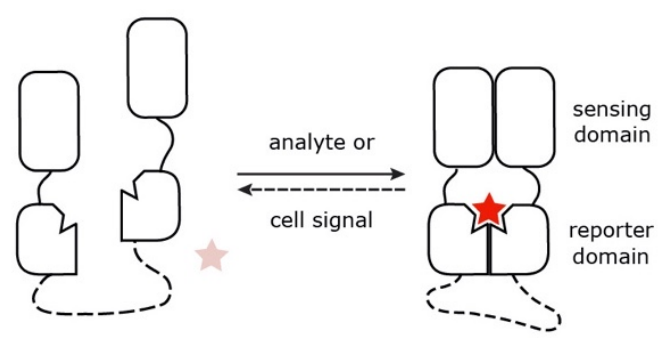

Chemists and biologists have recently built and breed a large collection of fluorogenic chemogenetic biosensors enabling to visualize and study cellular biochemistry in new ways. This review focuses on the new perspectives offered by fluorogenic reporters consistong in a protein or RNA tag that can complex either an endogenous or a synthetic fluorogenic dye (so-called fluorogen) to design unique and innovative biosensors, thus pushing the boundaries of cellular imaging. 